\title{
NOTIFICAÇÕES DE LEISHMANIOSE VISCERAL HUMANA: REALIDADE DO ESTADO DA PARAÍBA
}

\author{
HUMAN VISCERAL LEISHMANIASIS NOTIFICATIONS: REALITY OF \\ THE STATE OF PARAÍBA
}

\author{
Rosimery Cruz de Oliveira Dantas ${ }^{1}$ \\ Rosielly Cruz de Oliveira Dantas ${ }^{2}$ \\ Dilermando Simões Dantas ${ }^{3}$
}

RESUMO: Objetivou-se conhecer a tendência da leishmaniose visceral humana (LVH) no Estado da Paraíba e construir um perfil epidemiológico dos sujeitos acometidos. Adotou-se um estudo epidemiológico, série temporal, caráter quantitativo, com base nas notificações compulsórias de LVH, coletadas no site do Departamento de Informática do Sistema Único de Saúde. Teve como população as 1.027 notificações de leishmanioses no período de 2009 a 2018 e amostra 413 de LV. A análise foi realizada com estatística descritiva, utilizando-se do software Microsoft Excel 2013® e o SPSS versão 20.0. Os dados revelaram que a média de notificação na Paraíba, no período, foi 34,4 casos/ano, representando $1,1 \%$ da Região Nordeste e $0,71 \%$ do Brasil. Apresentou tendência oscilante no período estudado, no Brasil e na Paraíba. Mais prevalente em homens (64,9\%), na faixa etária $<5$ anos $(29,3 \%)$, com até 4 anos de estudo $(47,59 \%)$, da raça negra $(73,1 \%)$. $15,3 \%$ apresentou coinfecção com HIV, dos quais $52,4 \%$ teve cura e $7,9 \%$ foi a óbito. Conclui-se que o grupo etário de crianças e idosos é mais vulnerável. Ocorre mais em homens de baixa escolaridade e raça negra. A LVH se destaca como uma doença crônica e de alta letalidade quando não diagnóstica e tratada adequadamente e se caracteriza como um grave problema de saúde pública, por sua falta de controle, exigindo da Vigilância em Saúde, profissionais de saúde e gestores ações mais efetivas no rastreio da LV canina e controle dos animais de rua.

Palavras chave: Leishmaniose Visceral. Notificação Compulsória. Zoonose.

\footnotetext{
${ }^{1}$ Graduanda de Psicologia da Faculdade Santa Maria. Doutora em Saúde Coletiva. Docente da UFCG/CFP - Cajazeiras-PB.

${ }^{2}$ Graduanda de Enfermagem. UFCG/CFP - Cajazeiras-PB.

${ }^{3}$ Médico Veterinário. Doutorando do PPG de Medicina Veterinária - UFCG/CTRA - Patos PB.
} 
ABSTRACT: The objective was to know the tendency of human's visceral leishmaniasis (LVH) in Paraiba State and to build an epidemiological profile of the affected subjects. It was adopted an epidemiological study, time series, quantitative character, based on the compulsory notifications of $L V H$, collected on the website of the Departamento de Informação do Sistema Único de Saúde. Its population was 1,027 notifications of leishmaniasis in the period from 2009 to 2018 and the sample 413 from VL 413 from $L V$. The analysis was performed using descriptive statistics and the Microsoft Excel 2013® software and SPSS version 20.0. The data revealed that the average notification in Paraiba, in the period studied, was 34.4 cases/year, representing $1.1 \%$ of the Northeast Region and $0.71 \%$ of Brazil. It presented an oscillating trend in the period, in Brazil and Paraiba. Most prevalent in men (64.9\%), aged $<5$ years (29.3\%), with up to 4 years of study (47.59\%), black (73.1\%). $15.3 \%$ had coinfection with HIV, of which $52.4 \%$ had cure and $7.9 \%$ died. It is concluded that the age group of children and the elderly is more vulnerable. It occurs more in men with low education and black race. LVH stands out as a chronic disease with high lethality when not properly diagnosed and treated and is characterized as a serious public health problem, due to its lack of control, requiring health surveillance, health professionals and managers more effective actions in the field. canine LV screening and control of homeless animals.

Keywords: Visceral leishmaniasis. Compulsory notification. Zoonosis. 


\section{INTRODUÇÃO}

A relação do ser humano com animais vem de tempo antigos e traz inúmeros benefícios, sejam de ordem emocional, econômica ou social, que impactam diretamente na qualidade de vida do homem e do animal em si. Dentre os animais que mantêm maior convívio com o homem está o cão, com o qual é estabelecido forte vínculo afetivo. Domingues et al (2015), apontam que a interação entre homem e animal tende a reduzir os níveis de ansiedade e com isso diminui o índice de doenças relacionadas ao estresse. Lima e Grisotti (2018), afirmam que a estima por animais tem se acentuado de tal forma que na contemporaneidade são considerados membros da família e, até mesmo, substitutos de filhos e outros familiares, ampliando o fenômeno da antropomorfização. Dados da Pesquisa Nacional de Saúde (IBGE, 2015), aponta que 44,3\% dos domicílios possuem ao menos um cão, o equivalente a 28,9 milhões de residências.

Quando este convívio ocorre com animais que não estão com condições sanitárias adequadas, impacta negativamente na vida do homem, pois, pode ocasionar zoonoses. Segundo a Organização Mundial de Saúde (2016), zoonose é qualquer doença ou infecção naturalmente transmitida de animais vertebrados para humanos, causada por bactérias, vírus ou parasitas ou agentes não convencionais. Domingues et al (2015), destacam que a presença de animais de estimação em áreas públicas, tem aumentado o número de pessoas expostas ao risco de contrair zoonoses, que representam, em todo o mundo, cerca de $60 \%$ das doenças infecciosas que afetam os seres humanos e de $75 \%$ de doenças novas ou emergentes.

Dentre as zoonoses se destaca a Leishmaniose Visceral Humana (LVH), cujo cão é o principal reservatório, o que exige daqueles que mantem contato permanente com o animal a adoção de cuidados que caracteriza uma guarda responsável, como o uso de coleiras repelentes, vacinação, vermifugação, esterilização e manutenção de um ambiente adequado. Segundo a World Society for 
the Protection of Animals (WSPA), uma guarda responsável se traduz no cuidar da saúde física, psicológica e ambiental e do bem-estar do animal, bem como da prevenção de riscos à comunidade, no âmbito individual e coletivo (WHO, 2005). Felipetto (2018), destaca a necessidade de se promover políticas públicas de controle populacional animal, por meio de projetos educacionais e esterilização subsidiada para população de baixa renda.

A LVH é causada por protozoários heteroxênicos e intracelulares obrigatórios das espécies Leishmania chagasi ou L. infantum que afeta as células do sistema fagocítico mononuclear, é de notificação compulsória, pois apresenta elevado número de óbitos e está incluída entre as seis doenças endêmicas mais importantes do mundo (ALVES; FONSECA, 2018).

Segundo Leite (2016), a situação da leishmaniose no Brasil é um paradoxo: há o conhecimento sobre os aspectos mais importantes da doença, porém o seu controle ainda não foi conseguido. O que se observa é sua expansão geográfica, aumento da incidência e da sua letalidade. Ademais, há de se destacar que a sua ocorrência provoca prejuízos sociais e econômicos para o indivíduo, família, sociedade e cofres públicos. O tratamento recomendado pelo Ministério da Saúde (BRASIL, 2017), envolve a utilização de antibióticos (antimoniato de N-metil Glucamina como primeira escolha) e medidas de suporte como hidratação, uso de antitérmicos, hemoterapia e suporte nutricional. Além do monitoramento com a realização de exames laboratoriais e eletrocardiográficos para acompanhar a evolução do tratamento e identificar possível toxicidade medicamentosa.

Por ser uma doença que não possui barreiras geográficas e de alta letalidade quando não tratada, requer vigilância constante dos profissionais de saúde e todo caso suspeito deve ser submetido a investigação clínica, epidemiológica e aos métodos auxiliares de diagnóstico (BRASIL, 2017). Desta forma, a notificação assume papel imprescindível para gerar informações que direcionam as ações de saúde. Alvares et al (2015), destacam que a notificação necessita ser devidamente preenchida, pois, as informações sobre saúde são fontes valiosas de pesquisas, e se faz necessário vencer os vieses decorrentes da complexidade da prestação de cuidados e utilização dos serviços de saúde por parte dos usuários. 
Por isso, conhecer a realidade da LVH é imprescindível para o planejamento das ações de saúde, haja vista que cada espaço geográfico tem suas particularidades. Buscou-se, a partir das notificações da LVH, conhecer a realidade no Estado da Paraíba. Este estudo teve como objetivo conhecer a tendência da leishmaniose visceral humana no Estado da Paraíba, bem como construir um perfil epidemiológico dos sujeitos acometidos.

\section{MÉTODO}

Trata-se de um estudo epidemiológico do tipo série temporal, descritivo, de caráter quantitativo, realizado a partir da utilização de dados secundários, tendo como base as notificações compulsórias de LVH. A coleta de dados ocorreu no site do Departamento de Informática do Sistema Único de Saúde (DATASUS), cujos dados são de livre acesso. Para tanto se utilizou das informações em saúde (TABNET®), com as janelas: Epidemiológicas e morbidade, Doenças e agravos de notificação compulsória de 2007 em diante (SINAN), Leishmaniose visceral (LV), Estado da Paraíba, seguido das variáveis: ano e mês de notificação, Município de notificação, Escolaridade, Raça, Sexo, Faixa etária, Coinfecção HIV, que foram baixadas e agrupadas em tabelas geradas pelo software TABNET® para Windows 32, versão 3.0. A população do estudo compreendeu os 1.027 registros das notificações de LV e Tegumentar disponibilizadas no DataSUS no período de 2009 a 2018 e amostra todos os 413 de LV. Para melhor tabulação dos dados as variáveis foram agrupadas em categorias. A faixa etária em $>1$ ano, de 1 a 9, de 10 a 19, de 20 a 59, <60 anos; Raça/cor em Branca, Negra (preta/parda), Amarela/Indígena, Sem registro (ignorado/branco); A escolaridade em anos de estudo: Sem registro (branco/ignorado/não se aplica), nenhum ano de estudo, até 4 anos, de 5 a 8 anos, de 9 a 11 anos e $<12$ anos.

A análise dos dados foi realizada a partir de estatística descritiva, com frequências absolutas e relativas e medidas de tendência central, utilizando-se do software Microsoft Excel $2013 \circledR$, e apresentados sob a forma de gráfico e tabelas. 
Quanto aos aspectos éticos, mesmo se tratando de dados públicos, ressalta-se que foram fielmente observados e respeitados os itens dispostos nas Resoluções 466/2012 e 510/2016 do Conselho Nacional de Saúde.

\section{RESULTADOS E DISCUSSÃO}

No período compreendido de 2009 a 2018 foram realizadas 37.499 no Brasil, tendo a Região Nordeste com 20.145 (53.72\%) e o Estado da Paraíba com 413 notificações de $\mathrm{LVH}$, representando $1,1 \%$ do total brasileiro e $0,71 \%$, com média de 34,4 (desvio padrão $\pm 6,56$ ) de casos ano. Houve uma tendência oscilante, tanto no Brasil como na Paraíba, que retrata a clara falta de controle da doença (Gráfico 1). Houve registro em 30 municípios $(13,4 \%)$ dos 223 que compõe o território paraibano, sendo os que apresentaram maior registro: Campina Grande (139), João Pessoa (135), Cajazeiras (39), Sousa (32) e Patos (11). Considerando a população projetada pelo IBGE (2019), a maior prevalência foi do município de Cajazeiras com uma taxa de 60/1.000 habitantes, seguido de Sousa com 50/1.000 habitantes.

Para Brasil (2014), esta realidade decorre das características epidemiológicas e a insuficiência de conhecimentos sobre os diversos componentes da cadeia de transmissão da LV, e por isso, as estratégias de controle se efetivam em pequena escala. Segundo WHO (2010), para que ocorra um efetivo controle da LV, se faz um programa de controle da doença com ações integradas e, como os existentes ainda são raros, a morbimortalidade da leishmaniose mundial cresce de forma preocupante. 


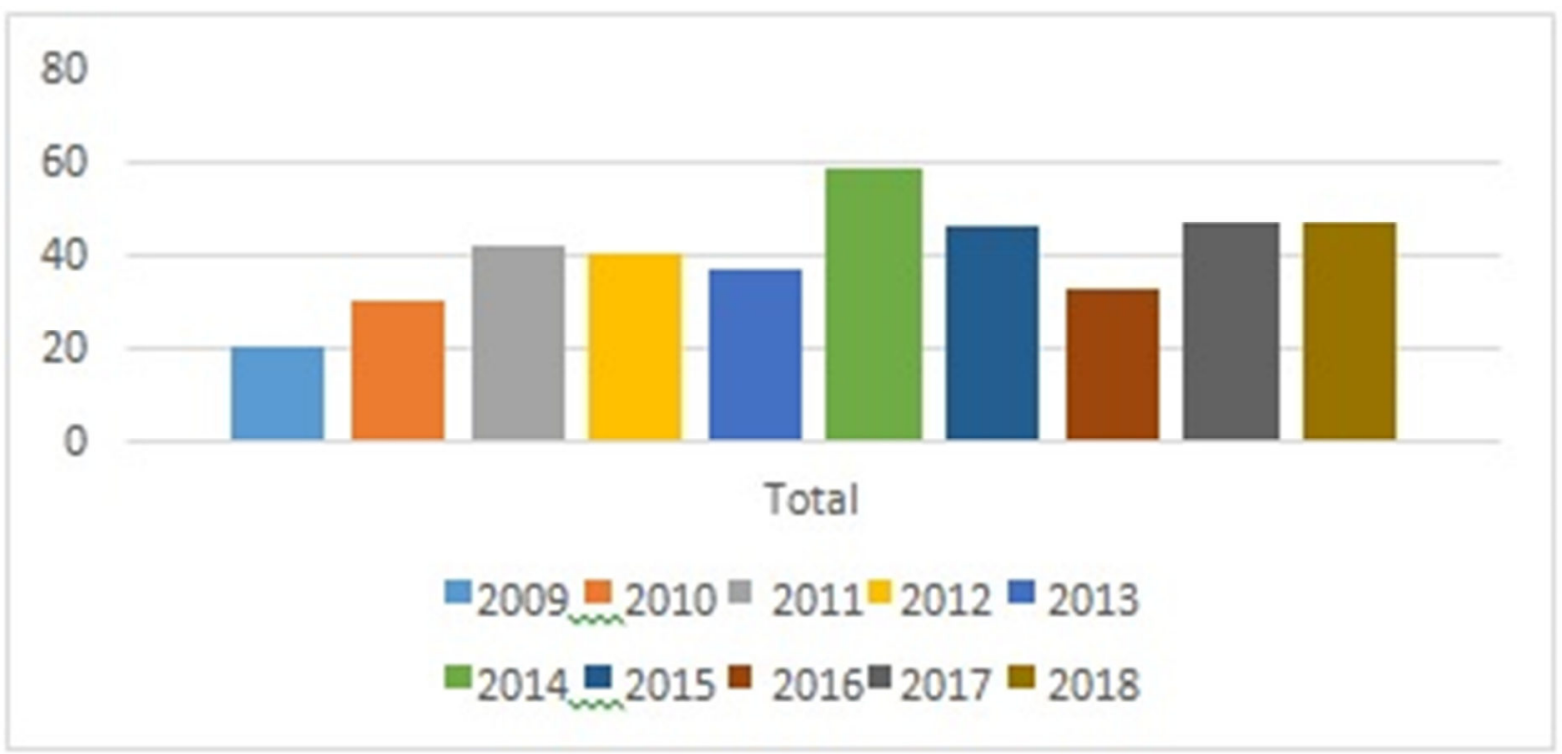

Gráfico 1 - Distribuições das notificações de LVH no Estado da Paraíba, 2009-2018.

Conforme disposto na Tabela 1, a maioria das notificações ocorreu no sexo masculino $(64,9 \%)$, na faixa etária $<5$ anos $(29,3 \%)$, de baixa escolaridade com até 4 anos de estudo $(47,59 \%)$, da raça negra $(73,1 \%)$, o que evidencia um grupo de maior exposição e vulnerabilidade. Destaca-se ainda que $15,3 \%$ apresentava coinfecção com HIV, dos quais $52,4 \%$ teve cura e $7,9 \%$ foi a óbito.

Com relação ao sexo, raça, faixa etária e escolaridade, este estudo corrobora com o de Leite (2016), realizado no Estado do Pernambuco, e o de Alves e Fonseca (2018), realizado em minas Gerais, trazendo evidências de que a LVH segue um padrão sociodemográfico. FELIPETTO (2018), destaca que baixo grau de escolaridade e baixa renda retrata o perfil das áreas de Estratégia de Saúde do Brasil e que, sujeitos com essas características, tendem a apresentar menos cuidados com a sanidade dos cães. Ademais, a expansão da LV para áreas urbanas e a manutenção de um ambiente propício, atrelado ao baixo nível socioeconômico e promiscuidade, bastante prevalente na zona rural e na periferia das cidades, tem favorecido a sua frequência em crianças menores de 10 anos e no sexo masculino (BRASIL, 2017).

Recomenda-se a oferta da sorologia para HIV para todos os pacientes com LV, independentemente da idade, uma vez que, o diagnóstico da coinfecção tem implicação na abordagem da LV no tocante ao diagnóstico, à indicação terapêutica e 
à ocorrência de recidivas. (Brasil, 2014). Por sua crescente distribuição geográfica, dificuldades no diagnóstico, no tratamento e altos índices de casos fatais, a coinfecção LV-HIVIAIDS pode ser considerada um problema emergente no Brasil (MARTINS-MELO et al., 2014).

Dado relevante, é o alto percentual de dados sem registro, que atingiu o patamar de $54,72 \%$ a $6,54 \%$. A ausência de dados faz com que o perfil epidemiológico traçado, caso houvesse a completude, apresentasse outro panorama e possibilitasse outra avaliação. Para Melo et (2018), essa realidade não contribui para as ações de vigilância, refletindo negativamente na vigilância epidemiológica.

Tabela 1 - Perfil epidemiológica das notificações de LVH no Estado da Paraíba, 2009-2018.

\begin{tabular}{cccc}
\hline \multicolumn{2}{c}{ Variável } & n & $\%$ \\
\hline Sexo & Feminino & 145 & 35,1 \\
& Masculino & 268 & 64,9 \\
& $>5$ anos & 121 & 29,3 \\
Faixa Etária & 5 a 14 & 52 & 12,6 \\
& 15 a 19 & 25 & 6,0 \\
& 20 a 59 & 187 & 45,3 \\
& 60 e + & 28 & 6,8 \\
& Sem registro & 226 & 54,7 \\
Anos de estudo & Nenhum & 31 & 7,5 \\
& Até 4 & 58 & 14,1 \\
& 5 a 8 & 67 & 16,2 \\
& 9 a 11 & 29 & 7,0 \\
& 12 e + & 2 & 0,5 \\
Raça/cor & Sem registro & 27 & 6,5 \\
& Branca & 77 & 18,7 \\
& Negra & 302 & 73,1 \\
& Amarelo/Indígena & 7 & 1,7 \\
Infecção HIV & Sem registro & 105 & 25,4 \\
& Sim & 63 & 15,3 \\
& Não & 245 & 59,3 \\
\hline
\end{tabular}

Fonte: DataSUS/ Pesquisa 2019.

De acordo com a apresentação dos dados na tabela 2, nota-se que $89,6 \%$ das notificações são de casos novos, diagnósticos confirmatórios com Teste positivo de imunofluorescência $(45,8 \%)$ e parasitário $(33,7 \%)$. Porém, não foram realizados 
diagnósticos laboratoriais em $47,2 \%$ e $55,2 \%$, respectivamente, dos casos notificados. Dos casos notificados $58,1 \%$ foram curados e $9,9 \%$ a óbito.

Tabela 2 - Caracterização dos casos notificados de LVH no Estado da Paraíba, 2009-2018.

\begin{tabular}{cccc}
\hline & Variável & $\mathrm{n}$ & $\%$ \\
\hline \multirow{3}{*}{ Entrada } & Sem registro & 15 & 3,6 \\
& Caso novo & 370 & 89,6 \\
& Recidiva & 22 & 5,3 \\
& Transferência & 6 & 1,5 \\
& Sem registro & 5 & 1,2 \\
& Positivo & 189 & 45,8 \\
& Negativo & 24 & 5,8 \\
Parasitário & 195 & 47,2 \\
& Não realizado & 5 & 1,2 \\
& Sem registro & 139 & 33,7 \\
& Positivo & 41 & 9,9 \\
& Negativo & 228 & 55,2 \\
& Não realizado & 85 & 20,6 \\
& Sem registro & 240 & 58,1 \\
& Cura & 6 & 1,5 \\
& Abandono & 41 & 9,9 \\
& Óbito LV & 8 & 1,9 \\
& Óbito outra causa & 33 & 8,0 \\
\hline
\end{tabular}

Fonte: DataSUS/ Pesquisa 2019.

A confirmação diagnóstica tem importância epidemiológica para a instituição do tratamento adequado, favorecendo a cura e prevenindo o óbito. Para Brasil (2017), devido a LVH apresentar um período de incubação que varia de 10 a 24 meses e se caracterizar como doença crônica e sistêmica que, quando não tratada, evolui para óbito em $90 \%$ dos casos, o diagnóstico precoce laboratorial deve ser instituído. Este é realizado pela imunofluorescência indireta, que busca a presença de anticorpos circulante, e pelo parasitológico, sendo o mais efetivo, pois busca de formas amastigotas do parasito, realizado em ambiente hospitalar e em condições cirúrgicas. 


\section{CONSIDERAÇÕES FINAIS}

No Brasil, sem distinção de Estado ou Região, a LV tem apresentado um comportamento oscilante no tempo, evidenciando a falta de controle deste agravo. $\mathrm{O}$ grupo de crianças e idosos se constitui o mais vulnerável para a ocorrência da LVH, sendo mais prevalente em homens e da raça negra.

Por seu caráter epidemiológico, a LVH se destaca como uma doença crônica e de alta letalidade quando não diagnóstica e tratada adequadamente e se caracteriza como um grave problema de saúde pública, o que exige da Vigilância em Saúde, Vigilâncias Sanitária, Epidemiológica e Ambiental, dos profissionais de saúde e dos gestores ações mais efetivas no tocante ao rastreio da LV canina e controle dos animais de rua. Ademais, requer da população a adoção da posse responsável. No tocante as ações da Estratégia de Saúde da Família, cabe aos profissionais maior atenção para o diagnóstico diferencial e as condições de vida/moradia do usuário, haja vista que, o diagnóstico precoce e a instituição adequada do tratamento aumentam as chances de cura e a sobrevida do sujeito acometido.

Como todo estudo de base pública, traz como limitações a restrição geográfica, a incompletude dos dados e a atualização permanente dos dados. Porém, estas limitações não diminui o poder das informações e abre espaço para reflexão sobre o preenchimento das notificações, como responsabilidade social e de saúde pública. 


\section{REFERÊNCIAS BIBLIOGRÁFICAS}

ALVES, W.A.; FONSECA, D.S. Leishmaniose visceral humana: estudo do perfil clínicoepidemiológico na região leste de Minas Gerais, Brasil. J. Health Biol Sci, v.6, n.2, p:133-9, 2018. Disponível em: http://docs.bvsalud.org/biblioref/2018/04/882658/artigoo-2-id-1764v6_n2.pdf. Acesso em 22. dez. 2019.

BRASIL. Leishmaniose visceral. In: Guia de Vigilância em Saúde. $2^{a}$ edição. Brasília: Editora do Ministério da Saúde, 2017. Acesso em 19 de dezembro de 2019. Disponível em: http://portalarquivos.saude.gov.br/images/pdf/2017/outubro/06/Volume-Unico-2017. Acesso em 21. dez. 2019.

Manual de Vigilância e Controle da Leishmaniose Visceral. $1^{\mathrm{a}}$ edição. $5^{\mathrm{a}}$ reimpressão. Brasília: Ministério da Saúde, 2014. Disponível em: http://bvsms.saude.gov.br/bvs/publicacoes/manual_vigilancia_controle_leishmaniose_visceral_1 edicao.pdf. Acesso em 21. dez. 2019.

CARVALHO, G.F.; MAYORGA, G.R.S. Zoonoses e posse responsável de animais domésticos: percepção do conhecimento dos alunos em escolas no município de Teresópolis- RJ. Revista da Jopic, v.1, n.1, 2016. Disponível em: http://www.unifeso.edu.br/editora/pdf/202-613-1PB.pdf. Acesso em 23. dez. 2019.

DOMINGUES, L.R. et al. Guarda responsável de animais de estimação na área urbana do município de Pelotas, RS, Brasil. Ciência \& Saúde Coletiva, v.1, p:185-92, 2015. Disponível em: http://www.scielo.br/pdf/csc/v20n1/1413-8123-csc-20-01-00185.pdf. Acesso em 21.dez. 2019.

FELIPETTO, L. G. Perfil populacional e sanitário de cães e gatos associado ao perfil socioeconômico dos proprietários em áreas assistidas por Estratégias de Saúde da Família. Dissertação (Mestrado). 59p. Universidade Federal de Santa Maria - RS, Santa Maria RS, $2018 . \quad$ Disponível em: http://coralx.ufsm.br/ppgmv/images/dissertacoes2018/Lais\%20felipetto.pdf. Acesso em 21. dez. 2019.

INSTITUTO BRASILEIRO DE GEOGRAFIA E ESTATÍSTICA (IBGE). Estimativa populacional. 2019. Disponível em: https://www.ibge.gov.br/cidades-e-estados/pb.html. Acesso em 21. dez. 2019. Acesso em 21.dez. 2019.

Pesquisa Nacional de Saúde: acesso e utilização dos serviços de saúde, acidentes e violências: Brasil, grandes regiões e unidades da federação. 2015. Disponível em: < http://biblioteca.ibge.gov.br/visualizacao/livros/liv94074.pdf>. Acesso em 19. dez. 2019. Acesso em 21. dez. 2019.

LEITE, C.E.A. Leishamniose Visceral Humana em Pernambuco: Epidemiologia e Gastos com Internações Hospitalares. Dissertação (mestrado profissionalizante). 60p. Universidade Federal do Pernambuco - UFPE, Recife-PE, 2016. Disponivel em: https://repositorio.ufpe.br/bitstream/123456789/17851/1/Leishmaniose\%20Visceral\%20Hum ana $\% 20$ em $\% 20$ Pernambuco $\% 20$ -

\%20epidemiologia\%20\%20e\%20gastos\%20com\%20interna\%C3\%A7\%C3\%B5es\%20hospita lares.pdf. Acesso em 26.dez. 2019.

LAMPERT, M. Benefícios da relação homem-animal. Monografia (Graduação). 24p. Universidade Federal do Rio Grande do Sul- UFRS, Porto Alegre-RS, 2014. Disponível em: https://lume.ufrgs.br/handle/10183/104881. Acesso em 21. dez. 2019. 
LIMA, A.S.; SOUZA, M.B. Os benefícios apresentados na utilização da terapia assistida por animais: revisão de literatura. Revista Saúde e Desenvolvimento, vol.12, n.10, 2018. Disponível

https://www.uninter.com/revistasaude/index.php/saudeDesenvolvimento/article/view/880.

Acesso em 22.dez. 2019.

LIMA, C.C.; GRISOTTI, M. Relação humano-animal e leishmaniose: repercussões no cotidiano de indivíduos inseridos em região endêmica. Saude soc. v.27, n.4, p: 1261-9, Oct-Dec 2018. Disponível em: http://www.scielo.br/scielo.php?script=sci_abstract\&pid=S0104$12902018000401261 \&$ Ing=pt\&nrm=iso. Acesso em 22.dez. 2019.

MELO, M.A.S. et al. Percepção dos profissionais de saúde sobre os fatores associados à subnotificação no Sistema de Nacional de Agravos de Notificação. Rev Adm Saúde, v.18, n.71, abr-jun, 2018. Disponivel em: http://www.cqh.org.br/ojs- 2.4.8/index.php/ras/article/view/104. Acesso em 21. dez. 2019.

MARTINS-MELO, F. R. et al. Epidemiological patterns of mortality due to visceral leishmaniasis and HIVIAIDS co-infection in Brazil, 2000-2011. Trans R Soc Trop Med Hyg, v. 108, n. 6, p. 338 - 47, April 2014. Disponível em: https://www.ncbi.nlm.nih.gov/pubmed/24706340. Acesso em 23.dez. 2019.

ORGANIZAÇÃO MUNDIAL DA SAÚDE (OMS). Zoonoses. Disponível em: http://www.who.int/topics/zoonoses/en/. Acesso em 17 de dezembro de 2019.

OKUMURA, R.S.A. Perfil epidemiológico de leishmaniose humana no estado da Paraíba (2010 a 2015). Monografia (Graduação). 39p. Universidade Federal da Paraíba - Medicina Veterinária, Areia-PB: $\quad$ UFPB/CCA, 2018.2 Disponível: https://repositorio.ufpb.br/jspui/bitstream/123456789/3755/1/RSAO14032018.pdf. Acesso em 21.dez. 2019.

SILVA, R.B.S. et al. Aspectos epidemiológicos da leishmaniose visceral canina na zona rural do semiárido paraibano e análise de técnicas de diagnóstico. Pesq. Vet. Bras. [online]. vol.36, n.7, p:625-29, 2016. Disponível em: http://www.scielo.br/scielo.php?pid=S0100736X2016000700625\&script=sci_abstract\&tlng=pt. Acesso em 26.dez. 2019.

ZANELLA, J.R.C. Zoonoses emergentes e reemergentes e sua importância para saúde e produção animal. Pesq. agropec. bras., Brasília, v.51, n.5, p.510-519, maio 2016. Disponível em: http://www.scielo.br/pdf/pab/v51n5/1678-3921-pab-51-05-00510.pdf. Acesso em 19.dez. 2019. Acesso em 21.dez. 2019.

WORLD HEALTH ORGANIZATION (WHO). The control of neglected zoonotic diseases. Geneva: WHO, WSPA; $2005 . \quad$ Disponível em: https://www.who.int/neglected_diseases/zoonoses/9789241508568/en/. Acesso em 19. dez. 2019. Acesso em 21.dez. $201 \overline{9}$. 